\title{
An Effective Ant-Colony Based Routing Algorithm for Mobile Ad-hoc Network
}

\author{
Yingzhuang Liu ${ }^{1}$, Hong Zhang ${ }^{1}$, Qiang $\mathrm{Ni}^{2}$, Zongyi Zhou ${ }^{1}$, Guangxi Zhu ${ }^{1}$ \\ ${ }^{1}$ Department of Electronics and Information Engineering, Huazhong University of Science and Technology, \\ Wuhan, China, 430074, Email: liuyz@mail.hust.edu.cn \\ ${ }^{2}$ Electronic \& Computer Engineering, Brunel University, UK
}

\begin{abstract}
An effective Ant-Colony based routing algorithm for mobile ad-hoc network is proposed in this paper. In this routing scheme, each path is marked by path grade, which is calculated from the combination of multiple constrained QoS parameters such as the time delay, packet loss rate and bandwidth, etc. packet routing is decided by the path grade and the queue buffer length of the node. The advantage of this scheme is that it can effectively improve the packet delivery ratio and reduce the end-to-end delay. The simulation results show that our proposed algorithm can improve the packet delivery ratio by $9 \%-22 \%$ and the end-to-end delay can be reduced by $14 \%-16 \%$ as compared with the conventional QAODV and ARA routing schemes.
\end{abstract}

\section{INTRODUCTION}

Mobile Ad hoc NETwork (MANET) is a self-organized and self-managed network. Effective routing algorithm is one of its key technologies due to its dynamic network topology [1]. Supporting Quality of Service (QoS) is particularly important and challenging for emerging multimedia applications. Wang $\mathrm{Z}$ proved that it is a NP-C problem if a QoS routing scheme contains at least two constrain parameters [2]. In recent years, thanks to the development of bionic optimization algorithms, it offers a potential to develop a practical solution for intelligent routing strategies for MANET. It was presented in [3] a MMAS (Max Min Ant System) algorithm, in which the upper and lower limits of the pheromone are set. Only the pheromone of the best path is increased to accelerate the convergence speed. In [4] an encouragement and castigation strategy is proposed, in which best paths are encouraged and worst paths are castigated. However, there are still some deficiencies in the above mentioned algorithms, such as frequent updating of the pheromone and low convergence rate, etc.

In order to improve the performances of slow constringency and preference to stagnancy of traditional ant colony algorithm based routing scheme, an effective ant-colony based routing algorithm for mobile ad hoc network which is based on multiple constrained QoS parameters, namely AMQRA, is proposed in this paper. In the routing process, the obtained paths were marked by path grade, which is depended on the delay, packet loss rate and bandwidth, etc. and it updates the pheromone when it response to the routing request which depended on the path grade and the queue buffer length of the node. These mechanisms can accelerate the constringency and avoid the network converge. Our simulation results show that the algorithm can effectively improve packet delivery ratio and reduce end-to-end delay

The rest of this paper is organized as follows. In Section II., the multi-parameter constrained QoS routing problem for the MANET is presented and modeled. AMQRA routing algorithm is proposed in Section III. Section IV. shows the simulation results. Section V. concludes the paper.

\section{MULTIPLE CONSTRAINED QoS}

\section{ROUTING PROTOCOL FOR MANET}

Let a mobile ad hoc network be represented by a graph $G(V, E)$, where $V$ is the vertices and $E$ is its edges. The path between the source node $S$ and the destination node $D$ is defined as $P(S, D)$. We assume that there is only one link between two nodes in the process of routing. In reality, there are various QoS requirements from multimedia applications. The main factors considered in our AMQRA routing scheme are listed as the following parameters:

Time delay of a path:

$$
P_{\text {delay }}=\sum_{\text {link } \in P} \operatorname{delay}(\text { link })+\sum_{\text {node } P} \operatorname{delay}(\text { node })
$$

Packet loss rate of a path:

$$
P_{\text {loss }}=1-\prod_{\text {link } \in P}(1-l(\text { link }))
$$

Bandwidth of a path:

$$
P_{\text {bandwidth }}=\min \{b(\text { link })\},
$$

Where, delay(link), l(link) and b(link) are the time delay, the packet loss rate and the bandwidth of a particular link respectively, and delay(node) is the time delay of a particular node, all these parameters can be obtained by the routers.

The routing cost is the function of the time delay, the packet loss and the bandwidth of the path, namely,

$$
P_{\text {cost }}=\operatorname{func}\left(P_{\text {delay }}, P_{\text {loss }}, P_{\text {bandwidth }}\right)
$$


The purpose of the AMQRA algorithm is to find a path $P(S, D)$ that can satisfy the following multiple requirements:

For the time delay:

$$
P_{\text {delay }} \leq T
$$

For the packet loss rate:

$$
P_{\text {loss }} \leq L \text {, }
$$

For the bandwidth:

$$
\frac{1}{P_{\text {bandwidth }}} \leq \frac{1}{B},
$$

For the cost:

$$
P_{\cos t}<C,
$$

where, $T, L, B$ and $C$ are the restrictions of time delay, packet loss rate, bandwidth and cost of path respectively.

\section{AMQRA ROUTING ALGORITHM}

Our proposed AMQRA routing algorithm consists of three parts: routing discovery, routing maintenance and route failure handling. They are described in detail respectively in the following. In the process of routing, pheromone is a key parameter of a path, it has close relation with the time delay, packet loss rate and the bandwidth of the path, etc. the more the pheromone of a path, the more important the path will be and its grade is higher.

\section{$A$. Routing Discovery}

The creation of new routes in the routing discovery phase needs two kinds of ants: forward ant (FANT) and backward ant (BANT), FANT collects network information, and BANT updates the pheromone in the path. When FANT is broadcast by the sender, the intermediate nodes dispose the FANT as shown in Fig.1:

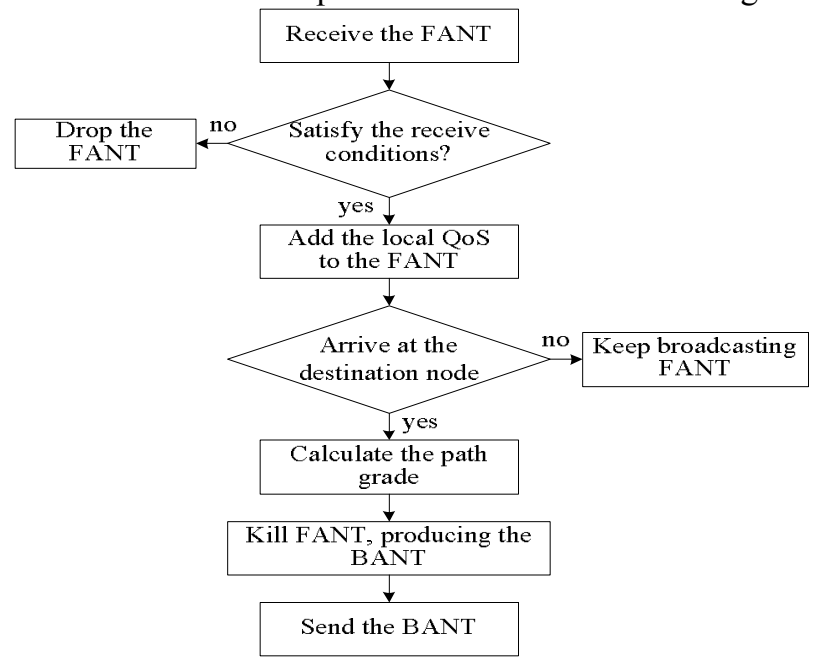

Fig.1: The process of FANT disposing
There are three restrictions for receiving a FANT in Fig.1:

(a) Satisfying the bandwidth requirement;

(b) The maximum hop number is limited by 6 ;

(c) Receiving the broadcast packet for the first time.

The node should satisfy the above three restrictions at the same time when it response to the FANT. In the process of routing discovery, the grade of path should be known, which depend on the cost of path. Generally, the more the time delay and the packet loss rate, the more the cost of the path would be. For the bandwidth, the more the bandwidth of the path, the less the cost of the path would be. So we define the cost of a path as:

$$
P_{\text {cost }}=\alpha P_{\text {delay }} \Omega\left(T-P_{\text {delay }}\right)+
$$

$$
\beta P_{\text {loss }} \Omega\left(L-P_{\text {loss }}\right)+\frac{\gamma}{P_{\text {bandwidth }}}
$$

where,

$$
\Omega(z)= \begin{cases}1 & \text { if } z \geq 0 \\ \infty & \text { if } z<0\end{cases}
$$

$\alpha, \beta$ and $\gamma$ are the normalized weights of time delay, packet loss rate and bandwidth respectively; which satisfies: $\alpha+\beta+\gamma=1$. And the value of $\alpha, \beta$ and $\gamma$ are determined by the service. For example, for the voice service, as the requirement of time delay is very rigorous, we should make $\alpha$ higher than $\beta$ and $\gamma$.

If $P_{\cos t}$ is infinite, it indicates that the QoS requirement of this path can not be met. The FANT will be killed. Otherwise, we will get its path grade $\rho$ by the following formula:

$$
\rho=e^{-P_{\cos t}}
$$

Then the intermediate node will dispose the BANT, as shown in Fig.2:

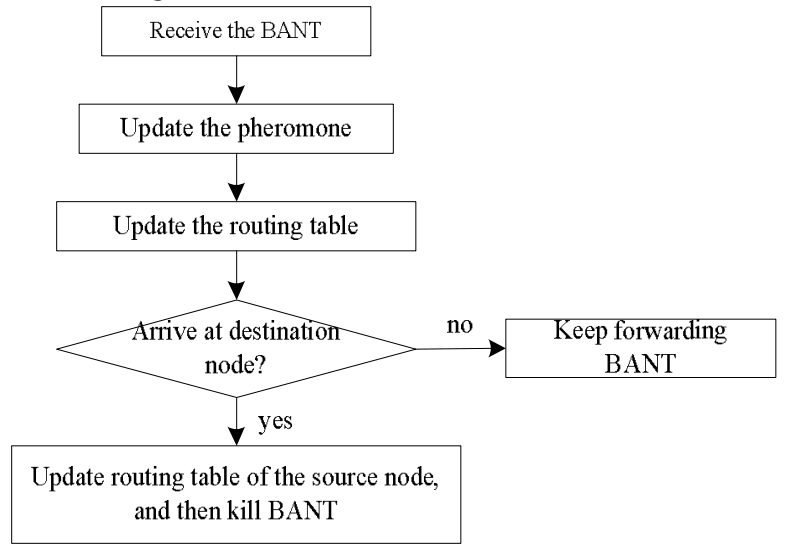

Fig.2: The process of the BANT disposing

The pheromone updating process in Fig. 2 is determined by the following formula:

$$
\tau_{v, u, s}=\tau_{v, u, s}+\Delta \tau_{v, u, s}
$$


where, $\tau_{v, u, s}$ is the pheromone of the link between nodes $v$ and $u$. $s$ is the source node, and at the beginning of the routing, $\tau_{v, u, s}$ is a const. for all $(v, u, s), \Delta \tau_{v, u, s}$ is its pheromone increment based on the cost of the path.

$$
\Delta \tau_{v, u, s}=\rho^{k} \quad(k \text { is an integer })
$$

The routing table is determined by $\tau_{v, u, s}$, when the source node receives a BANT, data packets will be sent by the path which has the highest grade.

\section{B. Routing Maintenance}

In our AMQRA scheme, the routing table is a probability table which is created according to the pheromone, as shown in Table1. Note that $D_{i}$ is the destination node. $N_{\mathrm{j}}$ is the neighbor node. $P_{\mathrm{ij}}$ is the probability of selecting $N_{\mathrm{j}}$ in the process of routing.

Table1: Probability routing table of AMQRA

\begin{tabular}{|c|c|c|c|c|}
\hline Neighbor node & $N_{1}$ & $N_{2}$ & $\ldots$ & $N_{k}$ \\
\hline$D_{1}$ & $P_{11}$ & $P_{12}$ & $\ldots$ & $P_{1 k}$ \\
\hline$D_{2}$ & $P_{21}$ & $P_{22}$ & $\ldots$ & $P_{2 k}$ \\
\hline$\ldots$ & $\ldots$ & $\ldots$ & $\ldots$ & $\ldots$ \\
\hline$D_{n}$ & $P_{n 1}$ & $P_{n 2}$ & $\ldots$ & $P_{n k}$ \\
\hline
\end{tabular}

The routing maintenance is composed of two parts: the updating of routing pheromone and the maintenance of neighbor nodes table, the updating of routing pheromone is determined by the following formula:

$$
\tau_{u, v, D}=(1-\rho) \tau_{u, v, D}+\psi e^{-k l}
$$

Where, $l$ is the queue buffer length of the node, $\psi$ is a coefficient, the first item in (14) is the reduced pheromone. The second item represents the increment part.

As the network topology and network flux changes with time, the routing table should be updated in a period of time $T$, we should send FANT to collect the information of network once more, and path grade should be modified according to Equation (14).

Neighbor nodes table is maintained by HELLO packets. If a node has never received HELLO packets within the expired time duration, it indicates that neighbor nodes are out of communication, the node should be deleted. If a node receives data packets, the probability of selecting a neighbor node $v$ is determined by the following formula:

$$
P_{u, v}=\frac{\left(\tau_{u, v, D}\right)^{\lambda}}{\sum_{v^{\prime} \in \operatorname{allow}(u)}\left(\tau_{u, v^{\prime}, D}\right)^{\lambda}}
$$

Where, $\lambda$ is a factor of heuristic information for the pheromone, allow $(u)$ is the set of neighbor nodes of $u$, which satisfy,

$$
\sum_{v^{\prime} \in \operatorname{allow}(u)} P_{u, v^{\prime}}=1
$$

In classical ant colony algorithm, the updating of pheromone includes both global updating and local updating, where the local updating process use local information. In AMQRA, the updating of pheromone is a local updating by global information. So it will reduce the end-to-end delay effectively.

\section{Route Failure Handling}

Due to the dynamic change of network topology caused by nodes random movement in MANET, routings often fail. Regarding the lost ACK of the link layer, nodes can detect the failure of the routing. When a node receives wrong messages, firstly we set the pheromone value to zero, and then search the routing table. If there are other routings to the destination node, data packets should be sent by the new routings. Otherwise we will send ERROR messages via inverse routing to inform upper nodes, and the upper nodes will delete error routings. If redundant routings exist, ERROR messages should be ceased. If there is no redundant routings in every node, the source node should launch a new route discovery process.

\section{SIMULATION RESULTS}

We employed 50 mobile nodes in the simulation, which can move in an area of $1500 \mathrm{~m} \times 300 \mathrm{~m}$. The maximal velocity of the node is set to be $20 \mathrm{~m} / \mathrm{s}$, the simulation time is $900 \mathrm{~s}$. The node mobility is expressed by the pause time and the max moving speed. We performed simulations with 10 different pause time 0,100 , 200, 300, 400, 500, 600, 700, 800 and 900 seconds.

Fig. 3 and Fig.4 are the simulation results of our proposed routing algorithm, AMQRA. In order to demonstrate its performance, we compare our results with the traditional QAODV (QoS based Ad hoc On-Demand Distance Vector Routing) and ARA (Ant-colony based Routing Algorithm). 


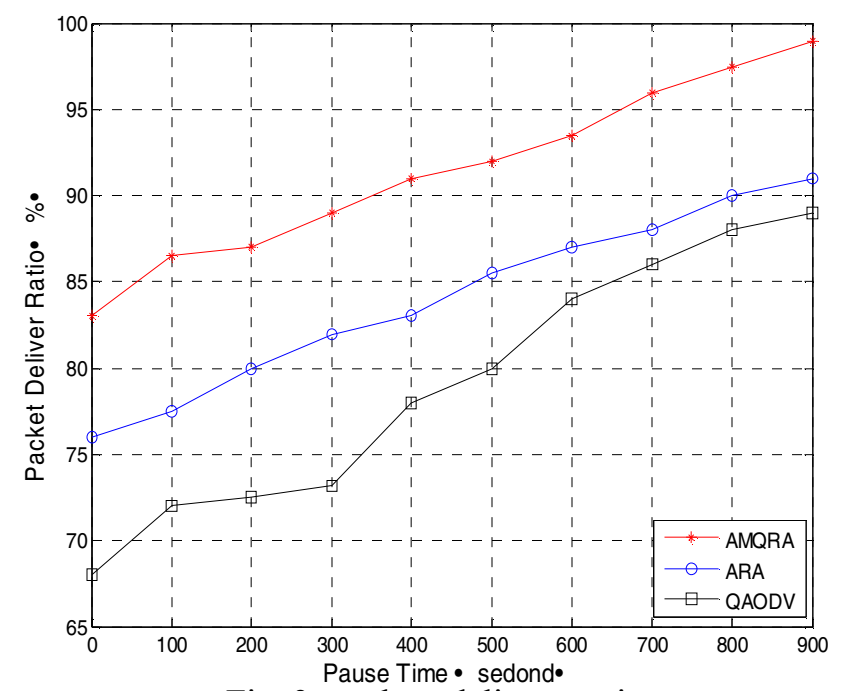

Fig. 3: packets delivery ration

Fig. 3 shows the packets delivery ratio of all routing protocol. We can see that AMQRA is higher than other two routing protocols by $9 \%-22 \%$ when the pause time is 0 , because AMQRA updates pheromone according to the network topology and QoS information. On the other hand it also updates pheromone by the queue length, which avoids network congestion. Therefore AMQRA has higher packets delivery ratio.

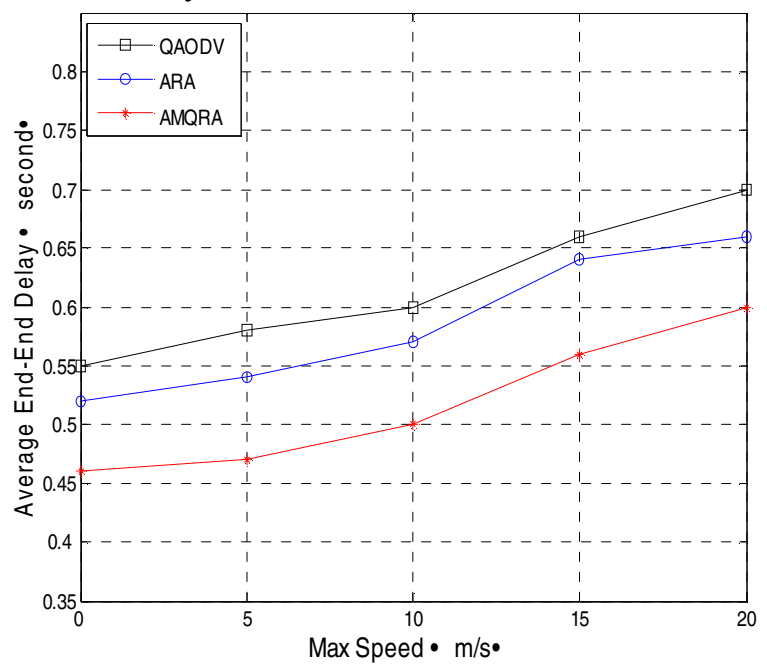

Fig.4: the average end-to-end delay

Fig.4 is the average end-to-end delay of all routing protocol. We can see that AMQRA has lower delay, because AMQRA updates pheromone by the queue length, the path which we choose is the least congestion one. It scouts the network's change all the time in order to reduce the probability of failure routing.

\section{CONCLUSIONS}

In this article, we present a novel multi-parameter constrained QoS routing scheme based on a new ant colony algorithm for mobile ad hoc network. Simulation results demonstrated that with our AMQRA scheme, the performances of packets delivery ratio and average end-to-end delay are superior to the traditional QAODV protocol and ant-colony based routing algorithm. Our future work will include applying our AMQRA scheme to the wireless network with various scales and node densities to test its performance, and to further improve the algorithm.

\section{ACKNOWLEDGEMENT}

This work is supported by the National 863 Projects (No. 2006AA01Z277)

\section{REFERENCES}

[1]. D.B.Johnson. "Routing in Ah Hoc network of mobile hosts". Proc. IEEE Workshop on mobile Computing Systems and Application, 1994

[2]. Z.Wang, J.Crowcroft. Quality of service routing for supporting multimedia applications [J]. IEEE Journal on Selected Areas in Communications, 1996

[3]. T. Stutzle, H.H. Hoos. MAX-MIN ant system and local search for the traveling salesman problem [C]. IEEE Int'Conf. on Evolutionary Computation, Indianapolis, IEEE Press, 1997: 309

[4]. M. Dorigo, V. Maniezzo, A. Colorni. The ant system: optimization by acolony of cooperating agents [J]. IEEE Transactionson Systems, Manand Cybernetics PartB, 1996, 26(1): 29-41

[5]. J. Broch, D. A. Maltz, D. B. Johnson, A performance comparison of multihop wireless ad hoc network routing protocols. Proceedings of the Fourth Annual ACM/IEEE International Conference on Mobile Computing and Networking (MobiCom' 98), 1998, pp. 85-97. 